\title{
Der Spezialist für Umweltschutz als ganzheitlicher Unternehmensmanager
}

\author{
Martin Lorko
}

\section{Einführung in die Problematik}

Das ständige Ansteigen des wissenschaftlich-ökonomischen Drucks auf kleine und mittlere Betriebe auf dem Gebiet des Umweltschutzes, der Produktionsqualität und der Arbeitssicherheit erfordert den Einsatz hoch qualifizierter, in diesen Bereichen allseitig ausgebildeter Ingenieure. Diese können den Druck nicht nur vermindern, sondern sollen ihn auch zum Vorteil ihrer Firma ausnutzen. Es geht im Folgenden um die Vorbereitung und den Einsatz der Ingenieure fuir Umweltschutz in kleinen und mittleren Unternehmen, die für die Lösung von Fragen im Zusammenhang mit der Steuerung der Umweltfuirsorge, der Gewährleistung der Produktionsqualität und der Beachtung verschiedener Risiken im Produktionsprozess zuständig sind. Diese Fachleute sollten außer einer gesunden Umwelt auch die Humanisierung der Arbeit und die Verbesserung der Arbeitsbedingungen im Produktionsprozess garantieren.

\section{Die fachliche Ausbildung der Umwelt- spezialisten in Unternehmen}

An erster Stelle sei hervorgehoben, dass wir keine neue Studiendisziplin als Ausdruck der Entfaltung einer neuen Art von Unternehmenstätigkeiten in der Slowakei definieren werden. Wir wollen über die entsprechende Ausbildung eines solchen Berufes sprechen, die das Leben selbst erfordert, weil ein wirksamer Umweltschutz, das Anwachsen der Produktionsqualität, die Sicherheit und der Gesundheitsschutz des Menschen heutzutage von oberster Priorität sind. Von der Hochschule wird heute gefordert, dass sie auf diesem Gebiet allseitig ausgebildete, hoch qualifizierte Fachkräfte für die Praxis vorbereitet. Diese sollen nicht nur als professionelle Schützer der Ökosysteme fungieren, auf die der Betrieb mit seinen Aktivitäten einwirkt, sondern gleichzeitig auch als Wächter über die Qualität der Betriebsproduktion und die Gesundheit seiner Arbeitskollegen.

Nach unserer Vorstellung ist der Umweltspezialist im Unternehmen (Generic Manager) ein vielseitiger Manager mit technischer Ausbildung, der die Grundprobleme bezüglich Umweltbildung und -schutz unter den Bedingungen der Praxis sowohl in technischer als auch juristischer Hinsicht lösen kann [5]. In kleinen und mittleren Unternehmen ist es vom ökonomischen Standpunkt her vorteilhaft und für die Sicherung verschiedener Spezialtätigkeiten perspektivisch sinnvoll, keine ausgeprägten Spezialisten, sondern allseitig ausgebildete Fachkräfte einzusetzen. Diese Fachleute können auch Tätigkeiten ausuiben, die mit Feuerschutz, Arbeits- und
Gesundheitsschutz, Zivilschutz, Qualitätsmanagement, mit der Humanisierung der Arbeit und der Arbeitsbedingungen sowie mit der Vermeidung von Abfällen und der Einsparung von Rohstoffen, Material, Wasser und Energie verbunden sind. Außerdem sind sie imstande, auch die fachliche Ausbildung der Mitarbeiter auf diesen Gebieten sicherzustellen [2].

Die Fachausbildung eines Spezialisten für Umweltschutz in einem Unternehmen muß einen breiteren Bildungsrahmen haben, auch zum Nachteil seiner Wissentiefe in diesen Bereichen. Diesen Mangel sollte er durch bessere Fachkommunikation mit den Spezialisten der einzelnen Gebiete ausgleichen. In der Vorbereitung des Umweltmanagers sollten insbesondere Kenntnisse zum Produktionsmanagement (ISO 9000 Quality management system), zu Systemen des Umweltschutzmanagement (ISO 14000 Environmental management system) und zur Risikosteuerung (Risk management) mit der Integration in ein Ganzes vermittelt werden. Im Hinblick auf seine breiten fachlichen Fähigkeiten ergeben sich geeignete Einsatzmöglichkeiten nicht nur in Klein- und Mittelbetrieben, sondern auch in der staatlichen und kommunalen Verwaltung, in der Kontrolle des Umweltzustandes, im Brandschutz sowie bei der Gewährleistung der Sicherheit der Bevölkerung. Nicht zuletzt kann er sein Wissen auch bei Beratungsfirmen, Banken oder Versicherungsanstalten anwenden [4].

\section{Diskussion (Meinungsaustausch)}

Die Analyse der Entwicklung der kleinen und mittleren Betriebe in der Slowakei zeigt, dass die Klein- und Mittelbetriebe im Jahre 1997 etwa $99 \%$ der Gesamtzahl der Unternehmen ausmachten. In diesen Betrieben sind zirka $60 \%$ der Gesamtbeschäftigten tätig. Sie erzeugen $58 \%$ der volkswirtschaftlichen Produktion der Slowakischen Republik [1].

Daraus ergibt sich, dass die Klein- und Mittelbetriebe in der Slowakei einen beträchtlichen Anteil am Beschäftigungsgrad und an der Bildung des Nationaleinkommens haben. Gleichzeitig aber werden sie zu einer potenziellen Bedrohung angesichts der Umweltverschmutzung und den damit verbundenen Beeinträchtigungen fuir das Leben und die Gesundheit der Bevölkerung. Es gilt vorläufig, dass mit steigendem Lebensstandard auch die Menge und Gefährlichkeit der Schadstoffe in der Umwelt ansteigt. Es gibt aber eine Grenze, die wir auf keinen Fall überschreiten dürfen, wenn wir die ständige Entwicklung der heutigen und natürlich auch zuküinftigen Generation sichern wollen. Im Interesse einer sinnvollen Nutzung der Umweltressourcen wird deshalb der 
Bedarf an fachlich hochqualifizierten Umweltingenieuren weiter zunehmen.

Untrennbarer Bestandteil des fachlichen Profils von Umweltspezialisten in Unternehmen ist auch ihre Befähigung zur Vorbereitung und Schulung der Mitarbeiter. Dazu gehört auch die Kommunikation mit den zuständigen Einrichtungen. Wir setzen voraus, dass die Eingliederung der Umweltspezialisten in die Praxis den Beschäftigungsgrad und die Konkurrenzfähigkeit der kleinen und mittleren Unternehmen in der Slowakei erhöht, zusammen mit der gleichzeitigen Verbesserung der Lebensqualität und Gesundheit der Mitarbeiter, also mit der Verbesserung der globalen ökologischen Bedingungen.

Umweltbildung und -schutz gehen uns alle an, das heißt, wir alle müssen uns der Lösung dieses Problems widmen - in unserem Lebensumfeld, am Arbeits- und Wohnort und überall dort, wo wir mit unseren Kenntnissen und Erfahrungen zur Wiederherstellung des Gleichgewichtes in den Ökosystemen beitragen. Jeder Absolvent der Hochschule muss eine umweltspezifische Ausbildung erhalten, vor allem diejenigen Absolventen, die auf ihrem Fachgebiet auch eine bestimmte Verantwortung für die Lösung der Umweltprobleme auf sich nehmen. Es gibt keine Hochschule, die solche Ökologen oder Umweltingenieure heranbilden könnte, die alle Probleme zu lösen imstande wären. Ein kompetenter Fachmann ist derjenige, der die Probleme auf dem Arbeitsplatz voraussehen kann und wirksame präventive Maßnahmen ergreift. Außerdem ist er sich über die Ursachen und Folgen der Umweltprobleme im Klaren und kennt auch die Wege für deren Beseitigung. Es genügt also nicht, eine schlechte Situation zu konstatieren und diese lediglich zu überwachen [2].

Wir können uns keine andere Lösung der Probleme in der Energiewirtschaft, im Maschinenbau, in der chemischen oder elektrotechnischen Industrie vorstellen, als die, dass technische Ingenieure, die die Ursachen und Möglichkeiten einer Lösung am besten kennen, sich ihrer annehmen. Wir behaupten nicht, dass in bestimmten Fällen nicht auch Fachkräfte aus den Bereichen Chemie, Biologie, Physik usw. solchen Arbeitsteams angehören sollten. Es gibt keinen Grund dafür, den Bedarf an Spezialisten für Ökologie und Umweltschutz über eine auf das Ressortprinzip beschränkte Ausbildung zu decken.

Es liegt auf der Hand, dass wirksame präventive und begleitende Maßnahmen zur Abwendung des Entweichens von Schadstoffen eher von demjenigen durchgefuihrt werden können, der die Produktionstechnologie in diesem Ressort kennt, als von demjenigen, der für dieses Gebiet nicht ausgebildet ist.

Im Hinblick auf die Umweltpolitik der Slowakei ist es erforderlich, in die konzeptionelle Planung auch die Legislative über die Funktion „Umweltspezialist im Unternehmen - Generic Manager" einzubeziehen, vor allem in den Bereichen, die durch den Charakter ihrer Produktion und Dienstleistung die Umwelt am meisten bedrohen. Das fachliche Fundament dieses Berufsprofils muss jedoch in einer Ausbildung liegen, die am besten zur Lösung der konkreten ökologischen und umweltspezifischen Praxisprobleme beitragen kann.

\section{Abschluss}

Wir kommen zu der Meinung, dass die Slowakei, die sich im Zentrum des ,verschmutzten“ Europas befindet, von allen Einwohnern und Einrichtungen ein ständiges und komplexes Interesse an der Lösung der dringenden Umweltfragen einfordern muss. Die wichtigste Rolle spielen dabei die Ausbildungsinstitutionen, die bei der Herausbildung des ökologischen Bewusstseins der Menschen bzw. bei der Beseitigung ihres ökologischen Analphabetismus am meisten helfen können. Jede Bestrebung, diese Aktivitäten aus persönlichen, zentralistischen oder gruppeninternen Gründen zu bremsen, schadet der Natur und somit auch dem Menschen.

An der Fakultät der Produktionstechnologien der Technischen Universität Košice mit Sitz in Prešov haben wir reiche Erfahrungen in der Erziehung und Ausbildung auf dem Gebiet des Umweltschutzes seitens der Unternehmen sammeln können, und dies schon in der dritten Generation. Wir sind davon überzeugt, dass unser Kapital - die Investition in die Ausbildung junger Ingenieure als Umweltspezialisten für Unternehmen - der Gesellschaft vielfach in Form einer sauberen Umwelt, gesunden Bevölkerung und besseren Lebensqualität zurückgegeben wird.

\section{Literaturverzeichnis}

[1] Lorko, M.; Šimon, A.: „Priekopnícke“ vzdelávanie z Prešova - odborná príprava na funkciu podnikového ekológa. Hospodárske noviny, č.103, 1997, s. 7.

[2] Lorko, M.; Pavlenko, S.: Vyučovanie v podmienkach praxe ako súčast' učebných osnov v rámci študijného zamerania Podniková ekológia. Zborník prednášok zo seminára TEMPUS JET 11011, Bratislava 1998.

[3] Lorko, M.; Gerák, J.: Odvetvoví environmentalisti sú vo výrobe žiadanejší. Enviromagazin, roč.3, č.1, 1998, s. 31.

[4] Lorko, M.; Pavlenko, S.: Právne a odborné relevancie na profiláciu všestranného manažéra vo funkcii podnikového ekológa. Zborník prednášok z vedeckej konferencie „Energetické a environmentálne problémy pri prevádzke budov“, TU Košice 1997.

[5] Scherlofsky, A.: Umweltmanagementsysteme, časopis Umwelt \& Gemeide, č. 4, St. Pölten, 1998.

\section{Autor}

\section{Martin Lorko \\ TU Košice}

Fakultät für Produktionstechnologien in Prešov Lehrstuhl für Management und Umweltschutz Plzenská 10, 08001 Prešov, Slowakische Republik Tel. +4219172 2828

Fax +42191773 3453 